\title{
Fontes e parcelamento do nitrogênio em híbridos de milho geneticamente modificados
}

\author{
Nitrogen sources and splitting in genetically modified maize hybrids \\ Gustavo Henrique Demari ${ }^{1}$, Ivan Ricardo Carvalho ${ }^{2}$, Vinicius Jardel Szareski ${ }^{2}$, Diego Nicolau \\ Follmann ${ }^{1}$, Velci Queiróz de Souza ${ }^{3} \&$ Claudir José Basso ${ }^{3}$ \\ ${ }^{1}$ Universidade Federal de Santa Maria, Santa Maria, RS, Brasil. \\ ${ }^{2}$ Universidade Federal de Pelotas, Pelotas, RS, Brasil. *Autor para correspondência: carvalho.irc@gmail.com. \\ ${ }^{3}$ Universidade Federal de Santa Maria, Frederico Westphalen, RS, Brasil.
}

Submissão: 10/08/2015 | Aceite: 13/06/2018

\begin{abstract}
RESUMO
Objetivou-se neste trabalho avaliar os efeitos do parcelamento e das fontes nitrogenadas aos parâmetros morfológicos e ao rendimento de grãos em híbridos de milho geneticamente modificados. Este estudo foi realizado nas safras agrícolas 2012/2013 e 2013/2014 em Tenente Portela, RS. Utilizou-se o delineamento de blocos ao acaso organizado em esquema fatorial $(2 \times 2 \times 11 \times 3)$ sendo, safras agrícolas $x$ híbridos de milho $x$ manejo de nitrogênio $x$ fontes nitrogenadas, dispostos em três repetições. $A$ análise de variância evidenciou interação entre todos os fatores via altura de planta, número de grãos por fileira. Interação significativa via safras agrícolas $x$ híbridos de milho $x$ manejo de nitrogênio para a altura de inserção da espiga e rendimento de grãos. Evidenciou-se interação safras agrícola $x$ manejo de nitrogênio $x$ fontes nitrogenadas, e híbridos de milho $x$ manejo de nitrogênio para a variável altura de inserção da espiga. A característica massa de mil grãos apontou interação via safras agrícolas $\mathrm{x}$ híbridos de milho. Os híbridos respondem de maneira similar ao manejo de nitrogênio, onde as diferentes fontes nitrogenadas demonstram comportamento semelhante para as variáveis estudadas, não havendo resposta ao rendimento de grãos. Os diferentes manejos de nitrogênio proporcionam efeitos positivos para as variáveis aferidas, principalmente quando estes foram realizados em estádios iniciais da cultura. A recomendação atual de parcelamento do nitrogênio na cultura do milho está adequada, porém para o híbrido DKB $250 \mathrm{VT} P R O 2^{\circledR}$, em déficit hídrico, o nitrogênio pode ser aplicado até o estádio vegetativo V8.
\end{abstract}

PALAVRAS-CHAVE: Zea mays, crescimento, manejo nutricional, rendimento de grãos.

\section{ABSTRACT}

This study sought to evaluate the effects of sources and splitting of nitrogen on the morphological parameters and yield of genetically modified maize hybrids. This study was conducted during 2012/2013 and 2013/2014 growing seasons in Tenente Portela, Rio Grande do Sul, Brazil. Randomized blocks design in factorial arrangement $(2 \times 2 \times 11 \times 3)$ were used for the growing seasons; seasons $\mathrm{x}$ maize hybrids $x$ nitrogen management $x$ nitrogen sources, with three replicates. Analysis of variance pointed to interaction between all factors through plant height and number of kernels per row. Significant interaction through season $x$ maize hybrids $x$ nitrogen management for ear insertion height and grain yield. The interaction seasons $x$ nitrogen management $x$ nitrogen sources, and maize hybrids $x$ nitrogen management can be highlighted for the variable ear insertion height. The characteristic thousand grain weight showed interaction through seasons $x$ maize hybrids. Maize hybrids respond similarly to nitrogen management, where different nitrogen sources show similar behavior for the studied variables, without response to grain yield. The different nitrogen management forms caused positive effects on the evaluated variables, especially when performed in the early stages of culture. The current recommendation of nitrogen splitting in maize is adequate, but for the hybrid DKB 250 VT PRO2 ® in water deficit, nitrogen can be applied until the V8 growth stage.

KEYWORDS: Zea mays, growth, nutrient management, yield.

\section{INTRODUÇÃO}

O milho é utilizado na alimentação humana e animal, com grande importância econômica devido ao 
valor nutricional de seus grãos. O nitrogênio caracteriza-se como nutriente exigido em maior quantidade, sendo necessário em média $20 \mathrm{~kg} \mathrm{ha}^{-1}$ deste nutriente por tonelada de grãos produzidos. Para altos rendimentos a cultura depende de oferta auxiliar de nitrogênio, devido ao solo não atender a demanda deste nutriente durante o desenvolvimento da cultura. As recomendações de nitrogênio para o milho no estado do Rio Grande do Sul e Santa Catarina são embasadas em apliçacões via semeadura e por cobertura durante os estádios vegetativos V4 e V6 (CQFS 2004).

Aplicações de nitrogênio via cobertura podem ser realizadas com a cultura entre quatro a oito folhas totalmente expandidas (DEMARI et al. 2016). As principais fontes nitrogenadas utilizadas para a cultura do milho correspondem à ureia e o sulfato de amônio. Segundo QUEIROZ et al. (2012), o sulfato de amônio apresenta liberação lenta do nitrogênio por ser recoberto por grânulos com substâncias orgânicas e inorgânicas, proporcionando a liberação gradual do nutriente para as plantas. Segundo VASCONCELLOS et al. (1998), a absorção do nitrogênio pelo milho é mais intensa entre 40 e 60 dias após a emergência das plântulas.

A disponibilidade de $\mathrm{N}$ na zona radicular é importante para garantir um adequado desenvolvimento inicial, resultando em acréscimos ao rendimento de grãos (VARVEL et al. 1997). Comportamento justificado através da definição do potencial produtivo do milho ocorrer em plantas com quatro a seis folhas totalmente expandidas (MAR et al. 2003). A assimilação do nitrogênio é variável em relação aos híbridos de milho, onde divergências genéticas influenciam diretamente na eficiência de assimilação e metabolismo deste nutriente (MACHADO et al. 1992, NARDINO et al. 2016). Alguns híbridos incorporam o íon amônio através de aminoácidos e estes potencializam a enzima glutamina sintase, resultando em acréscimos na eficácia do nitrogênio. Estudos de FERNANDES et al. (2005), demonstraram diferenças significativas aos caracteres morfológicos e produtivos de híbridos em relação ao aproveitamento do nitrogênio.

A eficiência no aproveitamento do nitrogênio pela cultura é intrinsecamente relacionada à época de aplicação, onde o parcelamento e a fonte de nitrogênio podem afetar alguns parâmetros morfológicos e influenciar o rendimento de grãos do milho. Portanto, objetivou-se neste trabalho avaliar os efeitos do parcelamento e das fontes nitrogenadas nos parâmetros morfológicos e no rendimento de grãos em híbridos de milho geneticamente modificados.

\section{MATERIAL E MÉTODOS}

Este estudo foi realizado em área de sucessão de culturas aveia-milho-aveia-milho, nas safras agrícolas 2012/2013 e 2013/2014 em Tenente Portela-RS, com altitude de 420 metros, Latitude de 27 $23^{\prime} 31$ 04"S e Longitude $53^{\circ} 46^{\prime} 5071^{\prime \prime} \mathrm{W}$. O clima é caracterizado segundo Köppen como subtropical úmido do tipo Cfa. O solo é classificado como Latossolo vermelho alumino férrico típico (STRECK et al. 2008). Por ocasião da instalação do experimento, foi realizada amostragem de solo na profundidade de 0-10 para a caracterização química que apresentou: argila: $64 \%$; pH em água: 6.0; índice SMP: 6,3; Fósforo (P): 4,3 mg $\mathrm{L}^{-1}$; Potássio (K): 159,5 mg L-1; Enxofre (S): 18,9 mg L-1; Matéria orgânica (MO): 2,9\%; Alumínio tóxico (Al):

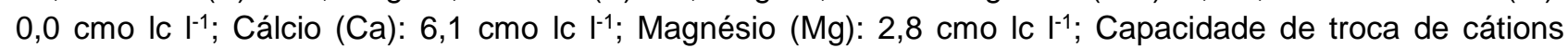

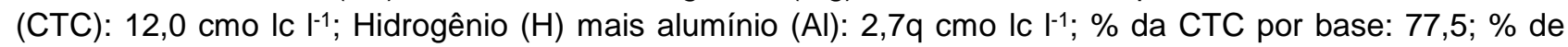
alumínio (Al): 0,0; Relação Cálcio (Ca)/magnésio (Mg): 2,2; Relação Cálcio (Ca)/potássio (K):15,0; Relação magnésio $(\mathrm{Mg}) /$ potássio(K): 6,9.

Utilizou-se o delineamento de blocos ao acaso organizado em esquema fatorial $(2 \times 2 \times 11 \times 3)$ sendo safras agrícolas $\mathrm{x}$ híbridos de milho $\mathrm{x}$ manejo de nitrogênio $\mathrm{x}$ fontes nitrogenadas, dispostos em três repetições. As unidades experimentais continham quatro linhas espaçadas por 0,5 metros com três metros de comprimento, totalizando seis metros quadrados, para as avaliações consideraram-se apenas as linhas centrais como parcela útil desconsiderando 0,25 metros iniciais, com intuito de minimizar os efeitos de bordadura.

Foram utilizados os híbridos simples de milho DKB 240 VT PRO2 ${ }^{\circledR}$ e DKB 250 VT PRO2. Os manejos de nitrogênio adotados foram: ausência de aplicação, aplicações totais em V2 (duas folhas totalmente expandidas), V4 (quatro folhas totalmente expandidas), V6 (seis folhas totalmente expandidas) e V8 (oito folhas totalmente expandidas), e parcelamento via V2+V4; V2+V6; V2+V8; V4+V6; V4+V8; V6+V8 e $\mathrm{V} 2+\mathrm{V} 4+\mathrm{V} 6+\mathrm{V} 8$. Todos os manejos com adição do nutriente totalizaram $200 \mathrm{~kg} \mathrm{ha}^{-1}$ de nitrogênio na forma de ureia e sulfato de amônio.

Para ambas as safras agrícolas utilizaram-se o sistema de semeadura direta realizada em 24/09/2012 e 03/10/2013 (Figura 1), com adubações na base de $450 \mathrm{~kg} \mathrm{ha}^{-1}$, na formulação (10-20-20) nitrogênio, fósforo e potássio, respectivamente. A população de plantas utilizadas para ambos os híbridos corresponde a 75.000 plantas por hectare e o controle de insetos-praga (Lufenurom) e doenças (Piraclostrobina + 
Epoxiconazol) foi realizado de maneira preventiva.
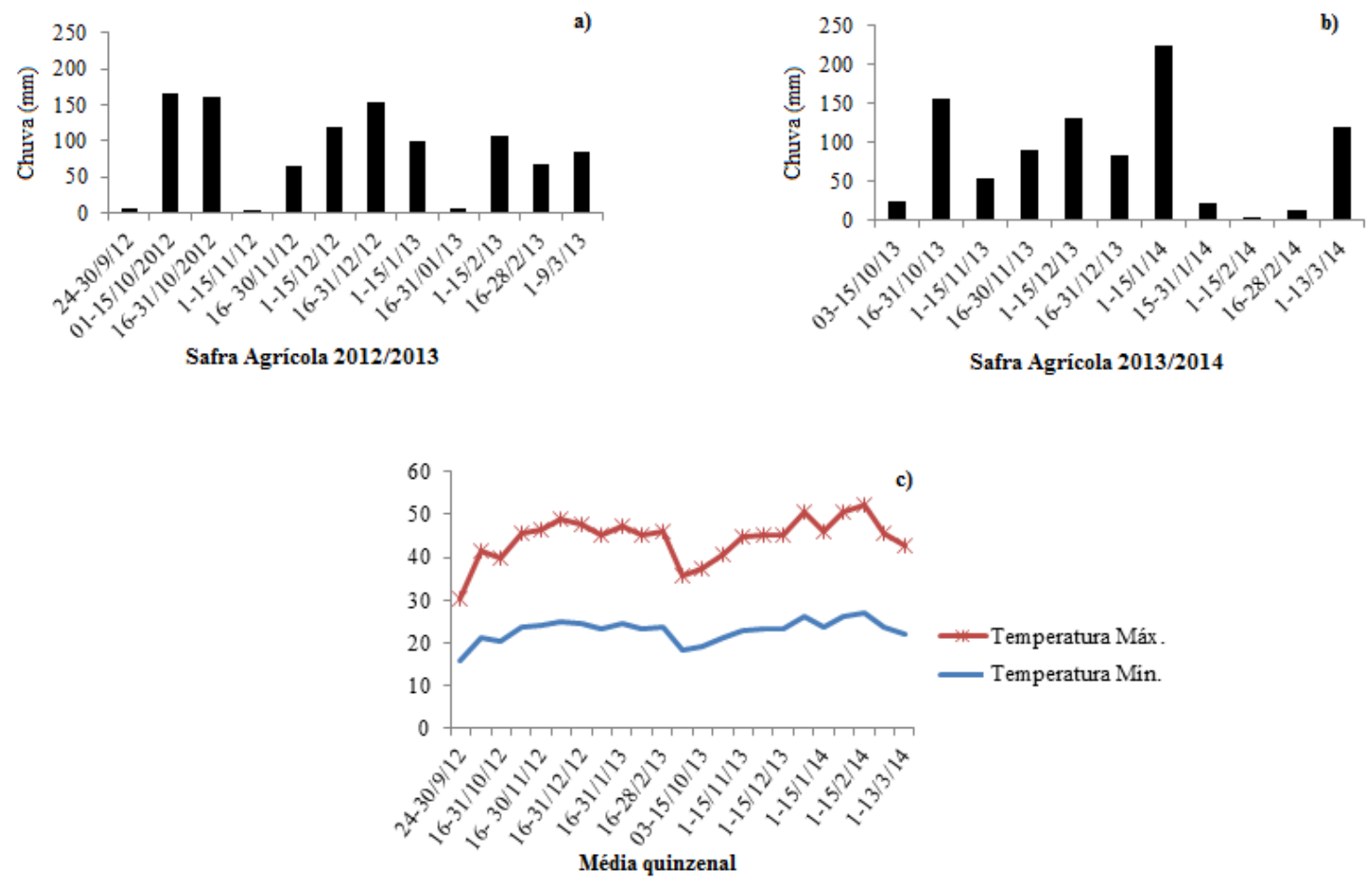

Figura 1. Precipitação acumulada por períodos quinzenais durante o período experimental na safra agrícola 2012/2013 (a) e safra agrícola 2013/2014 (b), e a média quinzenal das temperaturas mínimas e máximas no período de condução do experimento para ambas as safras agrícolas.

Figure 1. Bi-weekly cumulative rainfall during the 2012/2013 growing season (a) and 2013/2014 growing season (b) experimental periods, and the bi-weekly mean of the minimum and maximum temperatures during the experimental period for both growing seasons.

Os caracteres avaliados na ocasião da colheita foram altura de planta $(\mathrm{m})$, altura de inserção espiga (m), número de grãos por fileira, massa de grãos da espiga (g), massa de mil grãos (g), e rendimento de grãos $\left(\mathrm{kg} . \mathrm{ha}^{-1}\right)$. Os resultados obtidos foram submetidos à análise de variância pelo teste $\mathrm{F}$ e sendo significativo desmembraram-se aos efeitos simples das safras agrícolas $\mathrm{x}$ híbridos de milho $\mathrm{x}$ manejo de nitrogênio $x$ fontes nitrogenadas. As variáveis que não diferenciaram significativamente para interação foram comparadas o efeito principal via teste Tukey a $5 \%$ de probabilidade de erro $(p<0,05)$. Para confecção das análises utilizou-se o software estatístico Genes (CRUZ 2006).

\section{RESULTADOS E DISCUSSÃO}

A análise de variância evidenciou significâncias para interação safras agrícolas $x$ híbridos de milho $x$ manejo de nitrogênio $x$ fontes nitrogenadas para as variáveis altura de planta, número de grãos por fileira. Observou-se interação significativa entre safras agrícolas $\mathrm{x}$ híbridos de milho $\mathrm{x}$ manejo de nitrogênio para a altura de inserção da espiga e rendimento de grãos. Evidenciou interação safras agrícola $x$ manejo de nitrogênio $x$ fontes nitrogenadas, e híbridos de milho $x$ manejo de nitrogênio para a variável altura de inserção da espiga. $O$ caráter massa de mil grãos mostrou interação entre safras agrícolas $\mathrm{x}$ híbridos de milho.

A aplicação de ureia nos diferentes manejos, a altura de planta (Tabela 1) evidenciou semelhança para os híbridos de milho e safras agrícolas. O híbrido DKB 240 VT PRO2 ${ }^{\circledR}$ em ambas as safras agrícolas proporcionou maior altura de planta no parcelamento V2+V4. Para o híbrido DKB 250 VT PRO2 ${ }^{\circledR}$ na safra agrícola 2012/2013 observa-se maior altura de planta via aplicação de ureia, porém, na safra agrícola 2013/2014 este caráter evidencia-se superior em manejos de nitrogênio V2, V4, e V4+V6.

O sulfato de amônio para o híbrido DKB 240 VT PRO2 ${ }^{\circledR}$ na safra 2012/2013 expressa superioridade para altura de planta via manejos nitrogenadas realizados em V4. Para a safra agrícola 2013/2014 a maior altura de planta é expressa aos manejos realizados em V2, V2+V4, V4+V6, V4+V8, V6+V8. O híbrido DKB 250 VT PRO2 ${ }^{\circledR}$ em 2012/2013 apontou magnitude superior de altura de planta para manejos via V4, em contrapartida a safra agrícola 2013/2014 evidencia o parcelamento V6+V8 inferior aos demais manejos. 
Tabela 1. Médias para interação safras agrícolas $x$ híbridos de milho $x$ manejo de nitrogênio $x$ fontes nitrogenadas para a variável altura de planta $(m)$, Ureia (Ur), Sulfato de amônio (SA), Ausência de nitrogênio (Aus N).

Table 1. Means for the interaction seasons $x$ maize hybrids $x$ nitrogen management $x$ nitrogen sources for plant height (m), Urea (Ur), ammonium sulfate (AS), absence of nitrogen ( $A N)$.

\begin{tabular}{|c|c|c|c|c|c|c|c|c|c|c|c|c|}
\hline \multicolumn{13}{|c|}{ Safra Agrícola 2012/2013 } \\
\hline \multirow{2}{*}{$\begin{array}{c}\text { Manejos de } \\
\text { Nitrogênio }\end{array}$} & \multicolumn{6}{|c|}{ DKB 240 VT PRO2 ${ }^{\circledR}$} & \multicolumn{6}{|c|}{ DKB 250 VT PRO2 ${ }^{\circledR}$} \\
\hline & \multicolumn{2}{|r|}{ Ur } & \multicolumn{2}{|r|}{ SA } & \multicolumn{2}{|r|}{ Aus N } & \multicolumn{2}{|c|}{ Ur } & \multicolumn{2}{|c|}{ SA } & \multicolumn{2}{|c|}{ Aus N } \\
\hline V2 & 1,78 & abAallI & 1,73 & abAallI & 1,78 & abcAallI & 1,72 & abAallI & 1,59 & bAallI & 1,63 & aAallI \\
\hline V4 & 1,65 & bBallI & 1,84 & aAallI & 1,83 & abAallI & 1,62 & abAallI & 1,80 & aAalll & 1,71 & aAallI \\
\hline V6 & 1,81 & abAallI & 1,70 & abAallI & 1,47 & eBallI & 1,67 & abAallI & 1,67 & abAallI & 1,58 & aAallI \\
\hline V8 & 1,67 & abABallI & 1,73 & abAallI & 1,56 & deBalll & 1,66 & abAallI & 1,68 & abAallI & 1,63 & aAallI \\
\hline $\mathrm{V} 2+\mathrm{V} 4$ & 1,82 & aAallI & 1,76 & abAallI & 1,76 & abcAalll & 1,78 & aAallI & 1,76 & abAallI & 1,69 & aAallI \\
\hline $\mathrm{V} 2+\mathrm{V} 6$ & 1,72 & abAallI & 1,79 & abAallI & 1,67 & bcdAalll & 1,65 & abAallI & 1,66 & abAallI & 1,68 & aAallI \\
\hline $\mathrm{V} 2+\mathrm{V} 8$ & 1,74 & abABallI & 1,66 & bBallI & 1,87 & aAallI & 1,71 & abAallI & 1,63 & bAallI & 1,66 & $\mathrm{aA \beta III}$ \\
\hline $\mathrm{V} 4+\mathrm{V} 6$ & 1,73 & abAallI & 1,68 & abAallI & 1,64 & cdeAallI & 1,66 & abAallI & 1,73 & abAallI & 1,63 & aAallI \\
\hline V4+V8 & 1,71 & abAallI & 1,70 & abAallI & 1,72 & abcdAallI & 1,67 & abAallI & 1,66 & abAallI & 1,74 & aAallI \\
\hline $\mathrm{V} 6+\mathrm{V} 8$ & 1,71 & abAallI & 1,67 & abAallI & 1,68 & bcdAallI & 1,56 & bAallI & 1,63 & abAallI & 1,66 & aAallI \\
\hline $\mathrm{V} 2+\mathrm{V} 4+\mathrm{V} 6+\mathrm{V} 8$ & 1,80 & abAallI & 1,80 & abAallI & 1,65 & cdAallI & 1,73 & abAallI & 1,68 & abAallI & 1,66 & aAallI \\
\hline
\end{tabular}

Safra Agrícola 2013/2014

\begin{tabular}{|c|c|c|c|c|c|c|c|c|c|c|c|c|}
\hline \multirow{3}{*}{$\begin{array}{c}\text { Manejos de } \\
\text { Nitrogênio } \\
\text { V2 }\end{array}$} & \multicolumn{6}{|c|}{ DKB 240 VT PRO2 ${ }^{\circledR}$} & \multicolumn{6}{|c|}{ DKB 250 VT PRO2 ${ }^{\circledR}$} \\
\hline & \multicolumn{2}{|r|}{ Ur } & \multicolumn{2}{|r|}{ SA } & \multicolumn{2}{|r|}{ Aus $\mathrm{N}$} & \multicolumn{2}{|c|}{ Ur } & \multicolumn{2}{|r|}{ SA } & \multicolumn{2}{|c|}{ Aus N } \\
\hline & 2,46 & abABall & 2,56 & aAall & 2,33 & bcBßII & 2,59 & aAall & 2,61 & aAall & 2,53 & aAall \\
\hline V4 & 2,36 & bcAßII & 2,46 & abAall & 2,39 & bcAall & 2,61 & aAall & 2,59 & aAall & 2,53 & aAall \\
\hline V6 & 2,41 & abcAall & 2,19 & $\mathrm{cB} \beta \|$ & 2,51 & abAall & 2,56 & abAall & 2,62 & aAall & 2,56 & aAall \\
\hline V8 & 2,33 & bcBall & 2,39 & abBall & 2,63 & aAall & 2,50 & abAall & 2,53 & aAall & 2,58 & aAall \\
\hline $\mathrm{V} 2+\mathrm{V} 4$ & 2,56 & aAall & 2,55 & aAall & 2,51 & abAall & 2,44 & abAall & 2,55 & aAall & 2,43 & aAall \\
\hline $\mathrm{V} 2+\mathrm{V} 6$ & 2,28 & $c A \beta I I$ & 2,39 & abAall & 2,38 & bcAall & 2,54 & abAall & 2,49 & aAall & 2,54 & aAall \\
\hline $\mathrm{V} 2+\mathrm{V} 8$ & 2,38 & bcAall & 2,42 & abAall & 2,44 & abAall & 2,39 & bAall & 2,55 & aAall & 2,46 & aAall \\
\hline V4+V6 & 2,40 & $a b c A \beta I I$ & 2,56 & aAall & 2,46 & abAall & 2,59 & aAall & 2,58 & aAall & 2,60 & aAall \\
\hline V4+V8 & 2,27 & $c B \beta I I$ & 2,53 & aAall & 2,09 & $\mathrm{dC} \beta I \mathrm{I}$ & 2,53 & abAall & 2,62 & aAall & 2,61 & aAall \\
\hline V6+V8 & 2,24 & cBall & 2,59 & aAall & 2,37 & bcBall & 2,42 & abAall & 2,18 & $\mathrm{bB} \beta I \mathrm{I}$ & 2,44 & aAall \\
\hline $\mathrm{V} 2+\mathrm{V} 4+\mathrm{V} 6+\mathrm{V} 8$ & 2,28 & $\mathrm{cB \beta} I \mathrm{I}$ & 2,30 & bcABßII & 2,46 & abAall & 2,53 & abAall & 2,56 & aAall & 2,59 & aAall \\
\hline
\end{tabular}

Médias seguidas pela mesma letra minúscula na coluna para estádios fenológicos, letra maiúscula na linha para fontes nitrogenadas para cada híbrido dentro de cada safra agrícola, letras gregas na linha entre híbridos dentro das safras agrícolas, e número romano na linha para estádios fenológicos dos híbridos entre safras agrícolas não diferem estatisticamente a Tukey com 5\% de probabilidade de erro.

Magnitudes maiores para altura de planta foram obtidas em manejos de nitrogênio realizados nos estádios iniciais, com acréscimos em crescimento do sistema radicular e acúmulo de fitomassa na planta. Com efeitos significativos atrelados à produção de hormônios como as giberelinas, auxinas e citocininas, quando conjugadas potencializam o crescimento, divisão e expansão celular (MARSCHNER 1995). Nos estádios iniciais do milho a planta acumula $43 \%$ do nitrogênio que necessita para o desenvolvimento pleno. GROSS et al. (2006), evidenciaram que o parcelamento da ureia para o milho incrementa a altura de planta de forma diferenciada para híbridos, onde maiores alturas de planta são expressas ao parcelamento V4-V5 + V7-V8 em determinado genótipo, e para outros melhores resultados são evidenciados via V6-V7. Estudos conduzidos por VON PINHO et al. (2008), mostraram resultados satisfatórios para adubação nitrogenada via ureia para os parcelamentos V4-V5, V6-V7 e V7-V8. O híbrido DKB 240 VT PRO2 ${ }^{\circledR}$ em 2012/2013 evidenciou superioridade para o sulfato de amônio aos estádios V4, em 2013/2014 o estádio V6 a ureia apresenta-se superior. O híbrido DKB 250 VT $\mathrm{PRO}^{\circledR}$ em 2012/2013 evidenciou altura de planta similar entre fontes nitrogenadas.

A superioridade da altura de planta em resposta a algumas fontes é justificada através da melhor eficiência na utilização deste nutriente, absorção, e indiretamente através de acréscimos em clorofila, que intercepta e assimila a radiação fotossinteticamente ativa (GOES et al. 2013). Em contrapartida estudos de ZUCARELI et al. (2014), apontam que épocas de aplicação e fontes de nitrogênio em milho, afirma que este caráter é intrínseco ao genótipo. Estudos de SCHONINGER et al. (2012), evidenciam menor altura de plantas quando se utilizou o sulfato de amônio em relação ao uso de ureia.

A altura de inserção da espiga (Tabela 2) não expressa diferença significativa entre as fontes nitrogenadas para ambas as safras agrícolas. Estudos realizados por GOES et al. (2013), evidenciaram 
superioridade da altura de inserção de espiga com sulfato de amônio. A altura de inserção da espiga oscilou entre as safras agrícolas, observou-se em 2012/2013 a utilização de ureia via V2+V4 e V4+V6 retrataram maiores valores, porém em 2013/2014 as aplicações em V4 e V4+V6 obteve-se melhores resultados.

Tabela 2. Interação para safras agrícolas $x$ manejos de nitrogênio $x$ fontes nitrogenadas para a variável altura de inserção da espiga (m), Ureia (Ur), Sulfato de amônio (SA), Ausência de nitrogênio (Aus N). Tenente Portela, RS, 2015.

Table 2. Interaction for seasons $x$ nitrogen managements $x$ nitrogen sources for the variable ear insertion height $(m)$, Urea (Ur), ammonium sulfate $(A S)$, absence of nitrogen ( $A N)$.

\begin{tabular}{|c|c|c|c|c|c|c|c|c|c|c|c|}
\hline \multirow{2}{*}{ Manejos de Nitrogênio } & \multicolumn{5}{|c|}{ Safra Agrícola 2012/2013 } & \multicolumn{6}{|c|}{ Safra Agrícola 2013/2014 } \\
\hline & & Ur & & SA & Aus $\mathrm{N}$ & & Ur & & SA & & Aus $\mathrm{N}$ \\
\hline V2 & 1,37 & $a b c A \alpha$ & 1,37 & $a A \alpha$ & $1,38 a b c A \alpha$ & 1,36 & $a b c A a$ & 1,40 & $a b c A \alpha$ & 1,27 & $\mathrm{cA \alpha}$ \\
\hline V4 & 1,36 & $a b c A \alpha$ & 1,39 & $a A \alpha$ & 1,45 aAa & 1,40 & $a b A \alpha$ & 1,39 & $a b c A \alpha$ & 1,31 & $a b c A a$ \\
\hline V6 & 1,38 & $a b c A \alpha$ & 1,32 & $\mathrm{aABa}$ & $1,23 \mathrm{~dB} \beta$ & 1,38 & $a b c A a$ & 1,39 & $a b c A \alpha$ & 1,42 & $a b A \alpha$ \\
\hline V8 & 1,29 & $\mathrm{cAa}$ & 1,32 & $\mathrm{aAa}$ & $1,26 \mathrm{cdA} \alpha$ & 1,36 & $a b c A a$ & 1,28 & $\mathrm{cA \alpha}$ & 1,35 & $a b c A a$ \\
\hline $\mathrm{V} 2+\mathrm{V} 4$ & 1,45 & $\mathrm{aA \alpha}$ & 1,41 & $\mathrm{aAa}$ & $1,42 a b A \alpha$ & 1,36 & $a b c A \alpha$ & 1,41 & $a b c A \alpha$ & 1,44 & $a b A \alpha$ \\
\hline $\mathrm{V} 2+\mathrm{V} 6$ & 1,35 & $a b c A \alpha$ & 1,39 & $a A \alpha$ & 1,37 abcdAa & 1,36 & $a b c A \alpha$ & 1,34 & bcAa & 1,36 & $a b c A a$ \\
\hline $\mathrm{V} 2+\mathrm{V} 8$ & 1,38 & $a b c A a$ & 1,30 & $a A \alpha$ & 1,40 abAa & 1,35 & $\mathrm{bcAa}$ & 1,35 & bcAa & 1,35 & $a b c A \alpha$ \\
\hline $\mathrm{V} 4+\mathrm{V} 6$ & 1,45 & $\mathrm{aAa}$ & 1,33 & $a A \alpha$ & $1,39 a b c A a$ & 1,42 & $a b A \alpha$ & 1,47 & $a b A \alpha$ & 1,44 & $a A \alpha$ \\
\hline $\mathrm{V} 4+\mathrm{V} 8$ & 1,30 & $\mathrm{bcAa}$ & 1,33 & $\mathrm{aA} \beta$ & 1,41 abAa & 1,33 & $a b c B a$ & 1,50 & $\mathrm{aAa}$ & 1,29 & $\mathrm{bcBa}$ \\
\hline V6+V8 & 1,33 & $a b c A a$ & 1,34 & aAa & 1,33 abcdAa & 1,25 & $\mathrm{cA \alpha}$ & 1,26 & $\mathrm{cA \alpha}$ & 1,30 & abcAa \\
\hline $\mathrm{V} 2+\mathrm{V} 4+\mathrm{V} 6+\mathrm{V} 8$ & 1,43 & $a b A a$ & 1,38 & $\mathrm{aAa}$ & 1,30 bcdAa & 1,32 & $a b c A \alpha$ & 1,33 & $\mathrm{bcAa}$ & 1,39 & $a b c A a$ \\
\hline
\end{tabular}

Médias seguidas pela mesma letra minúscula na coluna para estádios fenológicos, mesma letra maiúscula na linha para fontes nitrogenadas dentro de cada safra agrícola, e mesma letra grega na linha para as fontes entre safras agrícolas não diferem estatisticamente a Tukey com $5 \%$ de probabilidade de erro.

Resultados são justificáveis por MAGALHÃES et al. (2003), que relatam a influência do nitrogênio na determinação do potencial produtivo de híbridos de milho e as características vinculadas a espiga. O sulfato de amônio em 2012/2013 não evidenciou divergências quanto à altura de inserção da espiga em relação aos estádios de aplicação. Em 2013/2014 a superioridade deste parâmetro foi evidenciada para o parcelamento $\mathrm{V} 4+\mathrm{V} 8$, resultados explicáveis através do recobrimento do sulfato de amônio com enxofre que propicia liberação lenta do nitrogênio solúvel (GIRALDI \& FILHO 2003).

A altura de inserção da espiga entre os manejos nitrogenados e entre híbridos (Tabela 3) o híbrido DKB 240 VT PRO2 ${ }^{\circledR}$ em ambas as safras agrícolas obteve superioridade via parcelamento V2+V4. O híbrido DKB 250 VT PRO2® na safra agrícola 2012/2013 evidenciou superioridade nos parcelamentos V4+V6, no entanto em 2013/2014 manejos via V4, V6, V2+V6, V4+V6, V4+V8 resultaram em maior altura de inserção de espiga. As adubações nitrogenadas realizadas no estádio fenológico V6 propiciaram maior altura de inserção da espiga, justificável através do sistema radicular estar pouco desenvolvido, e explorar pouco o solo. Esta necessidade é crescente à medida que os estádios fenológicos avançam, sendo de fundamental importância o aporte do nitrogênio para propiciar um rápido crescimento das plantas (OKUMURA et al. 2011).

A altura de inserção da espiga em relação aos manejos de nitrogênio entre os híbridos de milho (Tabela 4) o DKB 240 VT PRO2 ${ }^{\circledR}$ via parcelamento V2+V4 para aplicações de ureia evidenciou magnitude superior, no entanto com sulfato de amônio os melhores resultados são observados via V4+V6. O híbrido DKB 250 VT PRO2 ${ }^{\circledR}$ em ambas as fontes, os manejos de nitrogênio via V4 e V4+V6 apresentam-se superiores. Estudos conduzidos por CABEZAS \& PÁDUA (2007), relataram maior eficiência do nitrogênio aplicado na forma de sulfato de amônio quando comparado à ureia, com efeitos aos caracteres morfológicos do milho.

A altura de inserção de espiga caracteriza-se de extrema importância para a cultura do milho, onde a menor distância entre o nível do solo e a espiga, contribuem para o equilíbrio, com redução do tombamento das plantas, sendo este caráter extremamente influenciável através da população de plantas (KAPPES et al. 2009), adubação nitrogenada (SANTOS et al. 2010), teores de potássio e características do genótipo (MEIRA et al. 2009). 
Tabela 3. Médias para interação safras agrícolas $x$ manejos de nitrogênio $x$ híbridos de milho a variável altura de inserção da espiga (m).

Table 3. Means for the interaction seasons $x$ nitrogen managements $x$ maize hybrids for the variable ear insertion height $(m)$.

\begin{tabular}{|c|c|c|c|c|c|c|c|c|}
\hline \multirow[b]{2}{*}{ Manejos de Nitrogênio } & \multicolumn{4}{|c|}{ Safra Agrícola 2012/2013 } & \multicolumn{4}{|c|}{ Safra Agrícola 2013/2014 } \\
\hline & \multicolumn{2}{|c|}{$\begin{array}{l}\text { DKB 240VT } \\
\text { PRO2 }^{\circledR} \\
\end{array}$} & \multicolumn{2}{|c|}{$\begin{array}{c}\text { DKB } 250 \text { VT } \\
\text { PRO2 }^{\circledR} \\
\end{array}$} & \multicolumn{2}{|c|}{$\begin{array}{c}\text { DKB 240VT } \\
\text { PRO2 }^{\circledR} \\
\end{array}$} & \multicolumn{2}{|c|}{$\begin{array}{r}\text { DKB } 250 \\
\text { VT PRO2 } \\
\end{array}$} \\
\hline V2 & 1,38 & $a b c d A \alpha$ & 1,37 & bcAa & 1,26 & $\mathrm{bcdB} \beta$ & 1,43 & $a b A \alpha$ \\
\hline V4 & 1,39 & $a b A \alpha$ & 1,41 & $a b A \beta$ & 1,26 & bcdBa & 1,51 & $a A \alpha$ \\
\hline V6 & 1,29 & $\operatorname{cdA\alpha }$ & 1,33 & bcA $\beta$ & 1,27 & bcdBa & 1,51 & $\mathrm{aA \alpha}$ \\
\hline V8 & 1,23 & $\mathrm{dA \alpha}$ & 1,29 & $\mathrm{cA} \beta$ & 1,22 & $\mathrm{cdBa}$ & 1,43 & $a b A \alpha$ \\
\hline $\mathrm{V} 2+\mathrm{V} 4$ & 1,45 & $\mathrm{aA \alpha}$ & 1,40 & $a b A a$ & 1,39 & $\mathrm{aA \alpha}$ & 1,41 & $a b c A \alpha$ \\
\hline $\mathrm{V} 2+\mathrm{V} 6$ & 1,40 & $a b A \alpha$ & 1,34 & bcA $\beta$ & 1,19 & $\mathrm{~dB} \beta$ & 1,48 & $a A \alpha$ \\
\hline $\mathrm{V} 2+\mathrm{V} 8$ & 1,38 & $a b c d A \alpha$ & 1,34 & bcAa & 1,29 & bcAa & 1,38 & $\mathrm{bcAa}$ \\
\hline $\mathrm{V} 4+\mathrm{V} 6$ & 1,35 & $\mathrm{bcdBa}$ & 1,46 & $\mathrm{aA \alpha}$ & 1,36 & $\mathrm{abBa}$ & 1,52 & $\mathrm{aAa}$ \\
\hline $\mathrm{V} 4+\mathrm{V} 8$ & 1,35 & $\mathrm{bcdA \alpha}$ & 1,35 & bcA $\beta$ & 1,25 & $\mathrm{cdB} \beta$ & 1,49 & $\mathrm{aAa}$ \\
\hline $\mathrm{V} 6+\mathrm{V} 8$ & 1,34 & bcdAa & 1,33 & bcAa & 1,24 & $\operatorname{cd} A \beta$ & 1,31 & $\mathrm{cA \alpha}$ \\
\hline $\mathrm{V} 2+\mathrm{V} 4+\mathrm{V} 6+\mathrm{V} 8$ & 1,38 & $a b c A \alpha$ & 1,36 & bcAa & 1,27 & $\mathrm{bcdB} \beta$ & 1,44 & $a b A \alpha$ \\
\hline CV(\%) & & & & & & & & \\
\hline
\end{tabular}

Médias seguidas pela mesma letra minúscula na coluna para estádios fenológicos, mesma letra maiúscula na linha para híbridos de milho dentro de cada safra agrícola, e mesma letra grega na linha para as fontes entre safras agrícolas não diferem estatisticamente a Tukey com $5 \%$ de probabilidade de erro.

Observa-se na tabela 5 o número de grãos por fileira, a safra agrícola 2012/2013 o DKB 240 VT $\mathrm{PRO}^{\circledR}$ com ureia mostrou superioridade para $\mathrm{V} 2, \mathrm{~V} 8, \mathrm{~V} 2+\mathrm{V} 4, \mathrm{~V} 2+\mathrm{V} 6, \mathrm{~V} 2+\mathrm{V} 8, \mathrm{~V} 4+\mathrm{V} 8, \mathrm{~V} 6+\mathrm{V} 8$, e V2+V4+V6+V8, porém em 2013/2014 foi responsivo ao manejo de nitrogênio V2 e V4. O híbrido DKB 250 VT PRO2 ${ }^{\circledR}$ em 2012/2013 expressou maior número de grãos por fileiras através do uso de ureia parcelada em V6+V8, e para 2013/2014 são evidenciados via parcelamento V2+V4+V6+V8. Diferenças entre híbridos de milho são justificadas por SOUZA et al. (2013), através das condições do ambiente e as características intrínsecas de cada material genético. Os resultados obtidos diferem de SILVA et al. (2005), que não observam diferenciações quanto ao número de grãos por fileira em relação a doses de nitrogênio. FERNANDES et al. (2005), evidenciaram melhores resultados para este caráter através de aplicações de ureia via $V 6$ e $\mathrm{V} 8$.

Tabela 4. Médias para interação entre híbridos de milho $x$ manejos de nitrogênio $x$ fontes nitrogenadas para a variável altura de inserção da espiga $(m)$, Ureia (Ur), Sulfato de amônio (SA), Ausência de nitrogênio (Aus N).

Table 4. Means for the interaction maize hybrids $x$ nitrogen managements $x$ nitrogen sources for the variable ear insertion height (m), Urea (Ur), ammonium sulfate (AS), absence of nitrogen (AN).

\begin{tabular}{|c|c|c|c|c|c|c|c|c|c|c|c|}
\hline \multirow{2}{*}{ Manejos de nitrogênio } & \multicolumn{5}{|c|}{ DKB 240 VT PRO2 ${ }^{\circledR}$} & \multicolumn{6}{|c|}{ DKB 250 VT PRO2 ${ }^{\circledR}$} \\
\hline & \multicolumn{2}{|r|}{$\mathrm{Ur}$} & \multicolumn{2}{|r|}{$\mathrm{SA}$} & Aus. N & \multicolumn{2}{|c|}{$\overline{U r}$} & \multicolumn{2}{|r|}{$\overline{S A}$} & \multicolumn{2}{|c|}{ Aus. N } \\
\hline V2 & 1,31 & $\mathrm{bAa}$ & 1,36 & $a b c A \alpha$ & $1,30 \quad a b c d A a$ & 1,42 & $a b c A \alpha$ & 1,42 & $a b c A a$ & 1,36 & $\mathrm{bAa}$ \\
\hline V4 & 1,26 & $\mathrm{bA} \beta$ & 1,35 & $a b c A \alpha$ & 1,37 abAa & 1,50 & $\mathrm{aAa}$ & 1,43 & $a b A \alpha$ & 1,45 & $a b A a$ \\
\hline V6 & 1,36 & $a b A \alpha$ & 1,25 & $c A \beta$ & 1,26 bcdAa & 1,40 & $a b c A \alpha$ & 1,46 & $\mathrm{aAa}$ & 1,38 & $a b A a$ \\
\hline V8 & 1,29 & $\mathrm{bAa}$ & 1,30 & $\mathrm{bcA} \alpha$ & $1,19 \mathrm{dA} \beta$ & 1,35 & bcAa & 1,31 & $\mathrm{bcA} \alpha$ & 1,40 & $a b A a$ \\
\hline $\mathrm{V} 2+\mathrm{V} 4$ & 1,44 & $\mathrm{aAa}$ & 1,43 & $a b A \alpha$ & 1,39 abAa & 1,37 & $\mathrm{bcAa}$ & 1,39 & $a b c A \alpha$ & 1,46 & $a b A a$ \\
\hline $\mathrm{V} 2+\mathrm{V} 6$ & 1,25 & $b A \beta$ & 1,35 & $a b c A \alpha$ & 1,33 abcAa & 1,45 & $a b A a$ & 1,38 & $a b c A \alpha$ & 1,39 & $\mathrm{bAa}$ \\
\hline $\mathrm{V} 2+\mathrm{V} 8$ & 1,36 & $\mathrm{abABa}$ & 1,25 & $\mathrm{cB} \beta$ & 1,40 aAa & 1,32 & $\mathrm{cA \alpha}$ & 1,40 & $a b c A \alpha$ & 1,35 & $\mathrm{bAa}$ \\
\hline $\mathrm{V} 4+\mathrm{V} 6$ & 1,35 & $a b A \beta$ & 1,39 & aAa & 1,32 abcA $\beta$ & 1,52 & $\mathrm{aA \alpha}$ & 1,44 & $\mathrm{aA \alpha}$ & 1,51 & $\mathrm{aAa}$ \\
\hline $\mathrm{V} 4+\mathrm{V} 8$ & 1,30 & $\mathrm{bABa}$ & 1,37 & $a b A a$ & $1,23 \mathrm{cdB} \beta$ & 1,33 & $\mathrm{cBa}$ & 1,46 & $a A \alpha$ & 1,47 & $a b A a$ \\
\hline V6+V8 & 1,27 & $\mathrm{bAa}$ & 1,32 & $a b c A \alpha$ & 1,29 abcdAa & 1,32 & $\mathrm{cA \alpha}$ & 1,29 & $\mathrm{cA \alpha}$ & 1,35 & $\mathrm{BAa}$ \\
\hline $\mathrm{V} 2+\mathrm{V} 4+\mathrm{V} 6+\mathrm{V} 8$ & 1,32 & $\mathrm{bAa}$ & 1,34 & $a b c A a$ & 1,31 abcdAa & 1,42 & $a b c A \alpha$ & 1,38 & $a b c A \alpha$ & 1,37 & $\mathrm{BAa}$ \\
\hline $\mathrm{CV}(\%)$ & & & & & & & & & & & \\
\hline
\end{tabular}

Médias seguidas pela mesma letra minúscula na coluna para estádios fenológicos, mesma letra maiúscula na linha para fontes nitrogenadas dentro de cada híbrido, e mesma letra grega na linha para as fontes entre híbridos não diferem estatisticamente a Tukey com $5 \%$ de probabilidade de erro.

Observa-se na Tabela 5 o número de grãos por fileira, a safra agrícola 2012/2013 o DKB 240 VT $\mathrm{PRO}^{\circledR}{ }^{\circledR}$ com ureia mostrou superioridade para $\mathrm{V} 2, \mathrm{~V} 8, \mathrm{~V} 2+\mathrm{V} 4, \mathrm{~V} 2+\mathrm{V} 6, \mathrm{~V} 2+\mathrm{V} 8, \mathrm{~V} 4+\mathrm{V} 8, \mathrm{~V} 6+\mathrm{V} 8$, e 
V2+V4+V6+V8, porém em 2013/2014 foi responsivo ao manejo de nitrogênio V2 e V4. O híbrido DKB 250 VT PRO2 ${ }^{\circledR}$ em 2012/2013 expressou maior número de grãos por fileiras através do uso de ureia parcelada em V6+V8, e para 2013/2014 são evidenciados via parcelamento V2+V4+V6+V8. Diferenças entre híbridos de milho são justificadas por SOUZA et al. (2013), através das condições do ambiente e as características intrínsecas de cada material genético. Os resultados obtidos diferem de SILVA et al. (2005), que não observam diferenciações quanto ao número de grãos por fileira em relação a doses de nitrogênio. FERNANDES et al. (2005), evidenciaram melhores resultados para este caráter através de aplicações de ureia via V6 e V8.

Tabela 5. Médias para interação safras agrícolas $x$ manejos de nitrogênio $x$ híbridos de milho $x$ fontes de nitrogênio para a variável número de grãos por fileira, Ureia (Ur), Sulfato de amônio (SA), Ausência de nitrogênio (Aus $\mathrm{N}$ ).

Table 5. Means for the interaction seasons $x$ nitrogen managements $x$ maize hybrids $x$ nitrogen sources for the variable number of kernels per row, Urea (Ur), ammonium sulfate (AS), absence of nitrogen $(A N)$.

\begin{tabular}{|c|c|c|c|c|c|c|c|c|c|c|}
\hline \multicolumn{11}{|c|}{ Safra Agrícola 2012/2013 } \\
\hline \multirow{2}{*}{$\begin{array}{c}\text { Manejos de } \\
\text { Nitrogênio }\end{array}$} & \multicolumn{4}{|c|}{ DKB 240 VT PRO2 ${ }^{\circledR}$} & \multicolumn{6}{|c|}{ DKB 250 VT PRO2 ${ }^{\circledR}$} \\
\hline & \multicolumn{2}{|c|}{$\overline{U r}$} & SA & Aus $\mathrm{N}$ & \multicolumn{2}{|c|}{$\overline{U r}$} & \multicolumn{2}{|c|}{$\mathrm{SA}$} & \multicolumn{2}{|c|}{ Aus $\mathrm{N}$} \\
\hline V2 & 41,4 & aAall & 37,9 abAall & 38,5 bAall & 31,4 & $\mathrm{cB \beta II}$ & 34,5 & bBall & 39,8 a & oAall \\
\hline V4 & 38,4 & bAall & 36,1 bAall & 38,0 bAall & 35,9 & abAall & 39,2 & aAall & 37,9 a & ocdAall \\
\hline V6 & 38,5 & bAall & 40,3 abAall & 38,7 bAall & 36,3 & abAall & 39,0 & aAall & 37,7 a & ocdAall \\
\hline V8 & 39,5 & aAall & 38,7 abAall & 37,3 bAall & 34,3 & bcAßII & 37,2 & abAall & $35,3 \mathrm{c}$ & Aall \\
\hline $\mathrm{V} 2+\mathrm{V} 4$ & 41,4 & aAall & 39,3 abAall & 40,0 abAall & 38,2 & abAall & 38,9 & abAall & 40,3 a & Aall \\
\hline $\mathrm{V} 2+\mathrm{V} 6$ & 41,0 & aAall & 39,1 abAall & 37,7 bAall & 37,9 & abAall & 36,1 & abAall & 35,5 a & ocdAall \\
\hline $\mathrm{V} 2+\mathrm{V} 8$ & 39,4 & aAall & 38,2 abAall & 38,5 bAall & 35,8 & abcAall & 40,0 & aAall & 39,9 a & oAall \\
\hline V4+V6 & 34,5 & bBall & 39,9 abAall & 43,0 aAall & 38,1 & abAall & 37,0 & abAall & 39,3 a & pcAall \\
\hline V4+V8 & 41,1 & aAall & 39,4 abAall & 39,5 abAall & 37,8 & abAall & 38,7 & abAall & 39,1 a & ocdAall \\
\hline$V 6+V 8$ & 39,9 & aAall & 39,4 abAall & 38,0 bAall & 39,2 & aAall & 38,1 & abAall & $35,9 \mathrm{~b}$ & cdAall \\
\hline $\mathrm{V} 2+\mathrm{V} 4+\mathrm{V} 6+\mathrm{V} 8$ & 39,9 & aAall & 40,7 aAall & 40,1 abAall & 37,4 & abABall & 39,5 & aAall & $34,8 d$ & $3 \beta I I$ \\
\hline \multicolumn{11}{|c|}{ Safra Agrícola 2013/2014 } \\
\hline \multirow{2}{*}{$\begin{array}{c}\text { Manejos de } \\
\text { Nitrogênio }\end{array}$} & \multicolumn{4}{|c|}{ DKB 240 VT PRO2 ${ }^{\circledR}$} & \multicolumn{6}{|c|}{ DKB 250 VT PRO2 ${ }^{\circledR}$} \\
\hline & \multicolumn{2}{|c|}{$\overline{U r}$} & SA & Aus $\mathrm{N}$ & \multicolumn{2}{|c|}{ Ur } & \multicolumn{2}{|c|}{ SA } & \multicolumn{2}{|c|}{ Aus $\mathrm{N}$} \\
\hline V2 & 38,3 & aAall & 37,5 aAall & 38,4 aAall & 32,8 & abcdAßII & 35,5 & abcAall & 36, & abAall \\
\hline V4 & 38,4 & aAall & 36,1 aAall & 40,2 aAall & 36,2 & abAall & 33,4 & abcdAallI & $33, \subseteq$ & bcA $\beta I$ \\
\hline V6 & 37,8 & abAall & 38,5 aAall & 38,1 aAall & 32,1 & bcdAßII & 32,9 & $a b c d A \beta I I I$ & 35,2 & bcAall \\
\hline V8 & 34,0 & abAalll & 36,1 aAall & 38,3 aAall & 35,4 & abcAall & 37,0 & aAall & 33,3 & bcAall \\
\hline $\mathrm{V} 2+\mathrm{V} 4$ & 36,0 & abAall & 36,9 aAall & 36,1 aAall & 34,5 & abcAall & 33,2 & abcdAallI & 34, & bcAalll \\
\hline $\mathrm{V} 2+\mathrm{V} 6$ & 37,5 & abAalll & 38,1 aAall & 37,8 aAall & 32,2 & bcdAßIII & 32,5 & bcdAßII & 33,1 & bcAall \\
\hline V2+V8 & 33,9 & bAall & 37,4 aAall & 37,4 aAall & 34,2 & abcAall & 31,9 & cdAßIII & 35,5 & bcAall \\
\hline V4+V6 & 37,5 & bAall & 35,2 aAallI & 37,1 aAallI & 31,1 & cdBßIII & 36,5 & abAallI & 35,7 & bcAall \\
\hline V4+V8 & 38,2 & abAall & 35,9 aAall & 37,7 aAall & 35,9 & abAall & 30,4 & $\mathrm{~dB} \beta I I I$ & 31,0 & $\mathrm{cB \beta III}$ \\
\hline $\mathrm{V} 6+\mathrm{V} 8$ & 36,5 & abAall & 37,5 aAall & 38,4 aAall & 28,5 & $\mathrm{~dB} \beta I I I$ & 33,2 & abcdABallI & 34,7 & bcAall \\
\hline $\mathrm{V} 2+\mathrm{V} 4+\mathrm{V} 6+\mathrm{V} 8$ & 36,00 & abAall & 36,5 aAall & 37,00 aAall & 36,8 & aAall & 32,8 & abcdAallI & 35,8 & bcAall \\
\hline CV(\%) & & & & & 7,34 & & & & & \\
\hline
\end{tabular}

Médias seguidas pela mesma letra minúscula na coluna para estádios fenológicos, letra maiúscula na linha para fontes nitrogenadas para cada híbrido dentro de cada safra agrícola, letras grega na linha entre híbridos dentro das safras agrícolas, e número romano na linha para estádios fenológicos dos híbridos entre safras agrícolas não diferem estatisticamente a Tukey com $5 \%$ de probabilidade de erro.

O sulfato de amônio em 2012/2013 para o híbrido DKB 240 VT PRO2 ${ }^{\circledR}$ apresentou maior número de grãos por fileira em manejos de nitrogênio via $V 2+V 4+V 6+V 8$, no entanto em 2013/2014 observa-se similaridade entre os manejos. SOUZA et al. (2011), evidenciaram ausência de respostas ao sulfato de amônio quando utilizado em semeadura e via cobertura em V6. O híbrido DKB 250 VT PRO2 ${ }^{\circledR}$ em 2012/2013 apresentou maior número de grãos por fileira em manejo de nitrogênio via V4, V6, V2+V8, V2+V4+V6+V8, porém na safra agrícola 2013/2014 este parâmetro expressou superioridade através de 
aplicações em V8. Estudos de ZUCARELI et al. (2014), evidenciaram eficiência superior para o uso de sulfato de amônio em semeadura combinado a aplicações via cobertura.

Os efeitos das fontes nitrogenadas são semelhantes entre os híbridos para a variável número de grãos por fileira, onde o híbrido DKB 240 VT PRO2 ${ }^{\circledR}$ em 2013/2014 e DKB 250 VT PRO2 ${ }^{\circledR}$ em 2012/2013 foram similares. Para ambas as safras agrícolas os híbridos DKB 240 VT PRO2 ${ }^{\circledR}$ e DKB 250 VT PRO2 ${ }^{\circledR}$ via parcelamento $\mathrm{V} 4+\mathrm{V} 6$ demonstraram superioridade para o sulfato de amônio. As diferenciações entre híbridos são embasadas na eficiência de conversão do nitrogênio em biomassa para cada genótipo (CANCELLIER et al. 2011). Estudos de SCHIAVINATTI et al. (2011), relataram influências ao número de grãos por fileira via fontes nitrogenadas, híbridos de milho e safras agrícolas. O número de grãos por fileira está atrelado as características do genótipo (FERNANDES et al. 2005, VALDERRAMA et al. 2011), doses de nitrogênio (CARMO et al. 2012), tamanho da espiga (KAPPES et al. 2009) e fontes de nitrogênio (ZUCARELI et al. 2014).

A massa de grãos por espiga do híbrido DKB 240 VT PRO2 ${ }^{\circledR}$ apresentou-se similar em relação aos manejos de nitrogênio, no entanto o híbrido DKB 250 VT PRO $2^{\circledR}$ expressou diferença nas aplicações em V2 e V6 (Tabela 6). Entre híbridos, DKB 240 VT PRO2 ${ }^{\circledR}$ apresenta-se superior para manejos nitrogenados via $\mathrm{V} 2, \mathrm{~V} 6, \mathrm{~V} 2+\mathrm{V} 6$ e V4+V8. Este comportamento é justificado através das características do genótipo, redução do florescimento, menor intervalo para o enchimento de grãos (MAGALHÃES et al. 2003). A massa de grãos por espiga relacionou-se diretamente ao rendimento de grãos, e sua magnitude é influenciável através de aportes nutricionais, principalmente do nitrogênio, e população de plantas (MELO et al. 2011), safras agrícolas (SCHIAVINATTI et al. 2011), fontes e parcelamento de nitrogênio (SORATTO et al. 2012).

Tabela 6. Médias para interação manejos de nitrogênio x híbrido de milho, para a variável massa de grãos da espiga (g).

Table 6. Means for the interaction nitrogen managements $x$ maize hybrids, for the variable weight of grains per cob $(g)$.

\begin{tabular}{ccc}
\hline Manejos de Nitrogênio & DKB 240 VT PRO2® & DKB250 VT PRO2 ${ }^{\circledR}$ \\
\hline V2 & $160,6 \mathrm{aA}$ & $138,5 \mathrm{bB}$ \\
V4 & $163,7 \mathrm{aA}$ & $150,8 \mathrm{abA}$ \\
V6 & $162,2 \mathrm{aA}$ & $140,9 \mathrm{bB}$ \\
V8 & $159,9 \mathrm{aA}$ & $150,9 \mathrm{abA}$ \\
V2+V4 & $150,3 \mathrm{aA}$ & $156,7 \mathrm{aA}$ \\
V2+V6 & $161,4 \mathrm{aA}$ & $146,5 \mathrm{abB}$ \\
V2+V8 & $158,8 \mathrm{aA}$ & $156,8 \mathrm{aA}$ \\
V4+V6 & $160,6 \mathrm{aA}$ & $158,4 \mathrm{aA}$ \\
V4+V8 & $163,7 \mathrm{aA}$ & 145,4 abB \\
V6+V8 & $157,8 \mathrm{aA}$ & $158,7 \mathrm{aA}$ \\
V2+V4+V6+V8 & $156,8 \mathrm{aA}$ & $161,1 \mathrm{aA}$ \\
\hline CV(\%) &
\end{tabular}

Médias seguidas pela mesma letra minúscula na coluna para estádios fenológicos, mesma letra maiúscula na linha entre híbridos não diferem estatisticamente a Tukey com $5 \%$ de probabilidade de erro.

A massa de mil grãos houve ausência de respostas significativas para o híbrido DKB 250 VT PRO2 ${ }^{\circledR}$ entre safras agrícolas (Tabela 7). O híbrido DKB 240 VT PRO2 ${ }^{\circledR}$ apresentou superioridade na safra agrícola 2012/2013. Em relação aos híbridos, em 2012/2013 o híbrido DKB 240 VT PRO2 ${ }^{\circledR}$ expressou superioridade, e para 2013/2014 os resultados foram inversos. Estudos de SCHONINGER et al. (2012), relataram que este caráter responde diretamente as características genéticas de cada híbrido. SILVA et al. (2005), apontaram grande interferência de fatores abióticos à massa de mil grãos. A massa de mil grãos sofre interferência do manejo nitrogenado, o que evidencia extrema importância ao rendimento da cultura (VELOSO et al. 2006), e sob o sistema de produção (CASTOLDI et al. 2011).

$A$ adubação nitrogenada influenciou o rendimento de grãos, onde na safra agrícola 2012/2013 (Tabela 8) o híbrido DKB 240 VT $\mathrm{PRO}^{\circledR}{ }^{\circledR}$ demonstrou superioridade nos manejos via V4, V2+V8, V4+V6 e $\mathrm{V} 2+\mathrm{V} 4+\mathrm{V} 6+\mathrm{V} 8$. O híbrido DKB $250 \mathrm{VT} \mathrm{PRO}^{\circledR}$ apresentou-se superior no manejo V4+V8. Para a safra agrícola 2013/2014 o DKB 240 VT PRO2 ${ }^{\circledR}$ não evidenciou respostas às aplicações de nitrogênio através de diferentes manejos, e o híbrido DKB $250 \mathrm{VT} P R O 2^{\circledR}$ expressou melhores respostas através de manejos em $\mathrm{V} 2, \mathrm{~V} 2+\mathrm{V} 4+\mathrm{V} 6+\mathrm{V} 8$. Os parcelamentos do nitrogênio propiciaram maior período de fornecimento deste nutriente para as plantas, e segundo MAGALHÃES et al. (2003), no estádio vegetativo V3 ocorre à definição do potencial produtivo do milho, em $\mathrm{V} 6$ o sistema radicular está em pleno funcionamento com alta taxa de 
absorção do nitrogênio, e em V8 o número de fileiras por espiga é definido. Estudos de SCHIAVINATTI et al. (2011), relataram que no estádio V8 apresentaram melhores respostas a eficiência do uso de nitrogênio pelo milho. Em contrapartida, SORATTO et al. (2012), não observaram diferenças significativas no rendimento de grãos via parcelamento do nitrogênio. Estudos de CARDOSO et al. (2011), concluíram seus trabalhos de forma similar.

Tabela 7. Médias para a interação safras agrícolas $\mathrm{x}$ híbridos de milho, para a variável massa de mil grãos (g).

Table 7. Means for the interaction seasons $x$ maize hybrids for the variable thousand grain weight (g).

\begin{tabular}{ccc}
\hline Safras Agrícolas & DKB 240 VT PRO2 ${ }^{\circledR}$ & DKB 250 VT PRO2 $^{\circledR}$ \\
\hline $2012 / 2013$ & $296,1 \mathrm{aA}$ & $272,1 \mathrm{aB}$ \\
$2013 / 2014$ & $255,7 \mathrm{bB}$ & $270,8 \mathrm{aA}$ \\
\hline CV(\%) & & 9,27
\end{tabular}

Médias seguidas pela mesma letra minúscula na coluna para safras agrícolas, e letras maiúsculas na linha para híbridos não diferem estatisticamente a Tukey com $5 \%$ de probabilidade de erro.

A adubação nitrogenada influenciou o rendimento de grãos, onde na safra agrícola 2012/2013 (Tabela 8) o híbrido DKB 240 VT PRO2 ${ }^{\circledR}$ demonstrou superioridade nos manejos via V4, V2+V8, V4+V6 e $\mathrm{V} 2+\mathrm{V} 4+\mathrm{V} 6+\mathrm{V} 8$. O híbrido DKB $250 \mathrm{VT} \mathrm{PRO}^{\circledR}$ apresentou-se superior no manejo V4+V8. Para a safra agrícola 2013/2014 o DKB 240 VT PRO2 ${ }^{\circledR}$ não evidenciou respostas às aplicações de nitrogênio através de diferentes manejos, e o híbrido DKB 250 VT PRO $2^{\circledR}$ expressou melhores respostas através de manejos em $\mathrm{V} 2, \mathrm{~V} 2+\mathrm{V} 4+\mathrm{V} 6+\mathrm{V} 8$. Os parcelamentos do nitrogênio propiciaram maior período de fornecimento deste nutriente para as plantas, e segundo MAGALHÃES et al. (2003), no estádio vegetativo V3 ocorre à definição do potencial produtivo do milho, em V6 o sistema radicular está em pleno funcionamento com alta taxa de absorção do nitrogênio, e em V8 o número de fileiras por espiga é definido. Estudos de SCHIAVINATTI et al. (2011), relataram que no estádio V8 apresentaram melhores respostas a eficiência do uso de nitrogênio pelo milho. Em contrapartida, SORATTO et al. (2012), não observaram diferenças significativas no rendimento de grãos via parcelamento do nitrogênio. Estudos de CARDOSO et al. (2011), concluíram seus trabalhos de forma similar.

Tabela 8. Médias para interação safras agrícolas $\mathrm{x}$ manejos de nitrogênio $\mathrm{x}$ híbridos de milho para a variável rendimento de grãos $\left(\mathrm{kg} \mathrm{ha}^{-1}\right)$.

Table 8. Means for the interaction seasons $x$ nitrogen managements $x$ maize hybrids for the variable grain yield $\left(\mathrm{kg} \mathrm{ha}^{-1}\right)$.

\begin{tabular}{|c|c|c|c|c|}
\hline \multirow[b]{2}{*}{ Manejos de Nitrogênio } & \multicolumn{2}{|c|}{ Safra Agrícola 2012/2013 } & \multicolumn{2}{|c|}{ Safra Agrícola 2013/2014 } \\
\hline & $\begin{array}{c}\text { DKB } 240 \text { VT } \\
\text { PRO2 }^{\circledR} \\
\end{array}$ & $\begin{array}{c}\text { DKB } 250 \text { VT } \\
\text { PRO2 }^{\circledR} \\
\end{array}$ & $\begin{array}{c}\text { DKB } 240 \text { VT } \\
\text { PRO2 }{ }^{\circledR} \\
\end{array}$ & $\begin{array}{c}\text { DKB } 250 \text { VT } \\
\text { PRO2 }^{\circledR} \\
\end{array}$ \\
\hline & \multicolumn{4}{|c|}{ 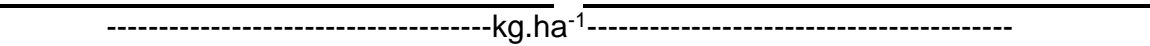 } \\
\hline V2 & $12450,8 \mathrm{abcAa}$ & $8962,6 \mathrm{fBa}$ & 6607,6 aA & 6563,1 aA $\beta$ \\
\hline V4 & 13615,1 aAa & 11734,7 cdeBa & 5974,0 aA $\beta$ & 6230,5 abA $\beta$ \\
\hline V6 & $11264,4 \mathrm{cAa}$ & 10927,4 deAa & 6483,5 aA $\beta$ & 5399,2 abA $\beta$ \\
\hline V8 & 11617,9 bcAa & 11538,5 cdeAa & 5652,8 aA $\beta$ & $4762,2 \mathrm{bA} \beta$ \\
\hline $\mathrm{V} 2+\mathrm{V} 4$ & 12868,6 abAa & $10565,1 \mathrm{eBa}$ & 5352,4 aA $\beta$ & 6023,0 abA $\beta$ \\
\hline $\mathrm{V} 2+\mathrm{V} 6$ & 12614,8 abcAa & 12224,4 bcdAa & 5798,5 aA $\beta$ & 5498,1 abA $\beta$ \\
\hline $\mathrm{V} 2+\mathrm{V} 8$ & 13205,3 aAa & 11898,2 cdeAa & 5710,6 aA $\beta$ & 5922,2 abA $\beta$ \\
\hline $\mathrm{V} 4+\mathrm{V} 6$ & 13360,7 aAa & $13645,3 \mathrm{abAa}$ & 5831,5 aA $\beta$ & 6284,8 abA $\beta$ \\
\hline $\mathrm{V} 4+\mathrm{V} 8$ & $12530,6 \mathrm{abcBa}$ & 14869,7 aAa & 5842,4 aA $\beta$ & 5471,2 abA $\beta$ \\
\hline $\mathrm{V} 6+\mathrm{V} 8$ & 12722,7 abcAa & 11276,9 cdeAa & 5545,6 aA $\beta$ & 5796,2 abA $\beta$ \\
\hline $\mathrm{V} 2+\mathrm{V} 4+\mathrm{V} 6+\mathrm{V} 8$ & 13207,3 aAa & $12515,5 \mathrm{bcAa}$ & 5755,7 aA $\beta$ & 6533,1 aA $\beta$ \\
\hline $\mathrm{CV}(\%)$ & \multicolumn{4}{|c|}{17,30} \\
\hline
\end{tabular}

Médias seguidas pela mesma letra minúscula na coluna para estádios fenológicos, mesma letra maiúscula na linha entre híbridos dentro de cada safra agrícola, e mesma letra grega na linha para híbridos entre safras agrícolas não diferem estatisticamente a Tukey com $5 \%$ de probabilidade de erro.

Ao comparar o comportamento entre híbridos, na safra 2012/2013 o híbrido DKB 240 VT PRO2 ${ }^{\circledR}$ evidenciou-se inferior ao DKB 250 VT $P R O 2^{\circledR}$ em aplicações via V4+V8, e comportamento inverso foram observados para DKB 240 VT PRO2 ${ }^{\circledR}$ que foi superior em aplicações via V2, V4, e parcelamento em V2+V4. 
Em 2013/2014 não se observou diferenças quanto às aplicações com diferentes manejos de nitrogênio. O rendimento de grãos apresentou-se superior para a safra agrícola 2012/2013 devido ao conjunto de condições climáticas favoráveis à cultura, tais como precipitação e temperatura ocorridas durante o período de condução do experimento. O rendimento de grãos apresenta contribuição de vários fatores, tais como densidade populacional, manejo nutricional (MELO et al. 2011), ambiente de cultivo (OKUMURA et al. 2011).

\section{CONCLUSÃo}

Os híbridos respondem de maneira semelhante ao manejo de nitrogênio, onde as diferentes fontes nitrogenadas demonstraram comportamento similar para as variáveis estudadas, não havendo resposta ao rendimento de grãos.

A recomendação atual de parcelamento do nitrogênio na cultura do milho está adequada. Contudo para o híbrido DKB 250VT PRO2®, em uma eventual condição de déficit hídrico, o nitrogênio pode ser aplicado até o estádio vegetativo V8, de modo a promover o incremento do rendimento de grãos.

\section{REFERÊNCIAS}

CABEZAS W \& PÁDUA R. 2007. Eficiência e distribuição de nitrogênio aplicado em cobertura na cultura de milho consorciada com Brachiaria ruziziensis, cultivada no sistema santa fé. Bragantia 66: 131-140.

CANCELLIER $L$ et al. 2011. Eficiência no uso de nitrogênio e correlação fenotípica em populações tropicais de milho no Tocantins. Revista Ciência Agronômica 42: 139-148.

CARDOSO SM et al. 2011. Fontes e parcelamento do nitrogênio em cobertura na cultura do milho sob plantio direto. Revista Brasileira de Ciências Agrárias 6: 23-28.

CARMO MS et al. 2012. Doses e fontes de nitrogênio no desenvolvimento e produtividade da cultura de milho doce (Zea mays var. Saccharata. rugosa). Bioscience Journal 28: 223-231.

CASTOLDI G et al. 2011. Sistemas de cultivo e uso de diferentes adubos na produção de silagem e grãos de milho. Acta Scientiarum. Agronomy 33: 139-146.

CQFS. 2004. Comissão de Química e Fertilidade do Solo - RS/SC. Manual de Adubação e de Calagem. Porto Alegre: SBCS. 394p.

CRUZ CD. 2006. Programa Genes - Estatística Experimental e Matrizes, $2^{a}$ Ed. Viçosa. 285p.

DEMARI G et al. 2016. Importance of nitrogen in maize production. International Journal of Current Research 8: 36629 36634 .

FERNANDES F et al. 2005. Doses, eficiência e uso de nitrogênio por seis cultivares de milho. Revista Brasileira de Milho e Sorgo 4: 195-204.

GIRALDI E \& FILHO F. 2003. Emprego de fertilizantes de liberação lenta na formação de pomares de citros. Laranja 24: 507-518.

GOES RJ et al. 2013. Características agronômicas e produtividade do milho sob fontes e doses de nitrogênio em cobertura no inverno. Revista Brasileira de Milho e Sorgo 12: 250-259.

GROSS M et al. 2006. Adubação nitrogenada, densidade de semeadura e espaçamento entre fileiras na cultura do milho em sistema de plantio direto. Ciência e Agrotecnologia 30: 387-393.

KAPPES $C$ et al. 2009. Influência do nitrogênio no desempenho produtivo do milho cultivado na segunda safra em sucessão à soja. Pesquisa Agropecuária Tropical 39: 251-259.

MACHADO A et al. 1992. Determinação da atividade de enzimas envolvidas no metabolismo do nitrogênio em diferentes genótipos de milho. Revista Brasileira Fisiologia Vegetal 4: 45-47.

MEIRA FA et al. 2009. Fontes e épocas de aplicação do nitrogênio na cultura do milho irrigado. Semina: Ciências Agrárias 30: 275-284.

MAGALHÃES PC et al. 2003. Fisiologia do Milho. Sete Lagoas: Embrapa. 23p. (Circular Técnica 22).

MAR G et al. 2003. Produção do milho safrinha em função de doses e épocas de aplicação de nitrogênio. Bragantia 62 : 267-274.

MARSCHNER H. 1995. Mineral nutrition of higher plants 2.ed. San Diego: Academic Press. 889p.

MELO FB et al. 2011. Fertilização nitrogenada, densidade de plantas e rendimento de milho cultivado no sistema plantio direto. Revista Ciência Agronômica 42: 27-31.

NARDINO M et al. 2016. Genetic parameters in maize hybrids analysis in different environments. International Journal of Current Research 8: 35552-35556.

OKUMURA RS et al. 2011. Uso de fertilizante nitrogenado na cultura do milho. Revista Brasileira de Tecnologia Aplicada nas Ciências Agrárias 4: 226-244.

QUEIROZ AM et al. 2012. Avaliação de diferentes fontes e doses de nitrogênio na adubação da cultura do milho (Zea mays L.). Revista Brasileira de Milho e Sorgo 10: 257-266.

SANTOS PA et al. 2010. Adubos verdes e adubação nitrogenada em cobertura no cultivo do milho. Revista Brasileira de Milho e Sorgo 9: 123-134.

SCHIAVINATTI AF et al. 2011. Influência de fontes e modos de aplicação de nitrogênio nos componentes da produção e produtividade do milho irrigado no cerrado. Bragantia 70: 925-930. 
SCHONINGER EL et al. 2012. Fontes e métodos de aplicação de nitrogênio na cultura do milho. Revista Agrarian 5: 365-372.

SILVA EC et al. 2005. Doses e épocas de aplicação de nitrogênio na cultura do milho em plantio direto sobre Latossolo Vermelho. Revista Brasileira Ciência do Solo 29: 353-362.

SORATTO RP et al. 2012. Parcelamento de fontes alternativas de nitrogênio no milho safrinha em sucessão à soja. Científica 40: 179-188.

SOUZA JA et al. 2011. Adubação nitrogenada na cultura do milho safrinha irrigado em plantio direto. Bragantia 70: 447454.

SOUZA RS et al. 2013. Produtividade e qualidade do milho doce em diferentes populações de plantas. Semina. Ciências Agrárias 34: 995-1010.

STRECK EV et al. 2008. Solos do Rio Grande do Sul. 2.ed. Porto Alegre: EMATER. 127p.

VARVEL GE et al. 1997. Ability for in-season correction of nitrogen deficiency in corn using chlorophyll meters. Soil Science Society of America Journal 61: 1233-1239.

VALDERRAMA $M$ et al. 2011. Fontes e doses de NPK em milho irrigado sob plantio direto. Pesquisa Agropecuária Tropical 41: 254-263.

VASCONCELLOS CA et al. 1998. Acúmulo de matéria seca e de nutrientes em milho cultivado no período invernoprimavera. Pesquisa Agropecuária Brasileira 33: 1835-1945.

VELOSO MEC et al. 2006. Doses de nitrogênio na cultura do milho, em solos de várzea, sob sistema de drenagem subterrânea. Revista Brasileira de Milho e Sorgo 5: 382-394.

VON PINHO RG et al. 2008. Adubação nitrogenada, densidade e espaçamento de híbridos de milho em sistema plantio direto na região sudeste do Tocantins. Bragantia 67: 733-739.

ZUCARELI C et al. 2014. Desempenho agronômico do milho safrinha em resposta às épocas de aplicações e fontes de nitrogênio. Científica 42: 60-67. 